\title{
A Tentative Observation Design for Investigating the Morphology of Dark Matter in the Universe
}

\author{
Jing Liu, Zijia Lin, Ziheng Shan \\ The High School Affiliated to Renmin University of China, Beijing, China \\ Correspondence to: Jing Liu, optimusprimewan@126.com; Zijia Lin, 13810514548@163.com; Ziheng Shan, \\ brianshan974@163.com
}

Keywords: Cosmology, Dark Matter, Gravitational Microlensing, Apparent Superluminal Motion

Received: September 28, $2020 \quad$ Accepted: November 16, $2020 \quad$ Published: November 19, 2020

Copyright $\odot 2020$ by author(s) and Scientific Research Publishing Inc.

This work is licensed under the Creative Commons Attribution International License (CC BY 4.0).

http://creativecommons.org/licenses/by/4.0/

\section{(c) (i) Open Access}

\section{ABSTRACT}

Unlike the luminous objects observed, dark matter does not emit light but can be only detected by its gravitational effect. Modern cosmology considers that most matter in Universe is dark matter. However, it is still not clear what the dark matter was. Two origins have been proposed by astrophysicists, astrophysics candidates and particle physics candidates. The most differences are their morphology, the former are compact objects and the latter are dispersed. Under Einstein's theory of general relativity, light bends as it passes near a compact object, creating a convergence effect like a lens. When background light source, intervening lense and the observer lie on a straight line, the brightness of the background source will be significantly magnified. In astrophysics, this effect is called microlensing. If compact dark matter is abundant in the universe, it is possible to frequently observe "microlensing" events when observing high redshift objects, i.e. the objects temporarily brighten for a certain time. The microlensing technique has been applied to study the dark matter in halo of Milky Way. The difficulty occurs when applying to study the cosmic dark matter as the crossing time of cosmic microlensing events is too long for observations. Apparent superluminal jets in bright quasars are idea background objects, significantly enhancing the efficiency of cosmic microlensing survey. Here, we tentatively designed an observational experiment to study the morphology of dark matter in Universe via statistics of microlensing events towards luminous quasars with apparent superluminal jets.

\section{INTRODUCTION}

\subsection{Discovery of Dark Matter}

In the 1930s, Fritz Zwicky, a Swiss astronomer at the California Institute of Technology, discovered something strange when he estimated the speed of galaxies within galaxy clusters using spectra. Galaxies 
move too fast relative to galaxy clusters. The gravitational field created by the summed mass of galaxies in a galaxy cluster simply cannot hold these galaxies together. Fritz estimated the mass of a galaxy cluster needed to hold the galaxies by the velocities of the galaxies, which is dozens of times greater than the summed mass of all the galaxies within the cluster. Therefore, Fritz named these invisible things "dark matter" [1]. Dark matter, however, has not attracted much attention due to the lack of other evidence.

In the 1960s, Vera Rubin and Kent Ford also noticed something strange when they were studying the rotation curves of Spiral Galaxy. During the measurement, they also found that the velocity of rotation of cold gas in stars and halos within the galaxies is too fast, which is known as the "rotation curve of galaxy" issue. As shown in Figure 1, there was a huge discrepancy in the galaxy peripheries when comparing the measured velocity with the theoretical rotation velocity estimated by the mass of stars.

As we know, given the altitude and orbital velocity of a satellite, the mass of the Earth can be derived from the law of gravity. Further, we can calculate the mass of the Sun using the revolution speed of the Earth and the distance between the Earth and the Sun. Therefore, we can predict the rotation curve of the galaxy through the mass of the glowing matter in the galaxy. It is most likely that the discrepancy between the mass of the glowing matter in the galaxy and the actual mass of the galaxy causes the deviation from the prediction. The image shows that the rotation velocity curve tends to be stable when the radius is very large, that is, the rotation velocity tends to be a constant. It is valid even when the distance from the center of the galaxy is huge. At the most periphery of a galaxy, the mass of glowing matter is very little. If the actual mass distribution of a galaxy is the same as that of the glowing matter, the rotation velocity in the galaxy periphery should be inversely proportional to the $1 / 2$ power of the radius. The velocity curve tends to be a constant means that the mass within a certain radius of the galaxy is proportional to the radius. The excessive mass over the glowing matter is called dark matter.

\subsection{Composition and Evolution of the Universe}

Currently, the prevailing perspective in cosmology is that the most dominant component of the universe is the so-called "dark energy". The 2011 Nobel Prize in Physics was awarded to Saul Perlmutter,

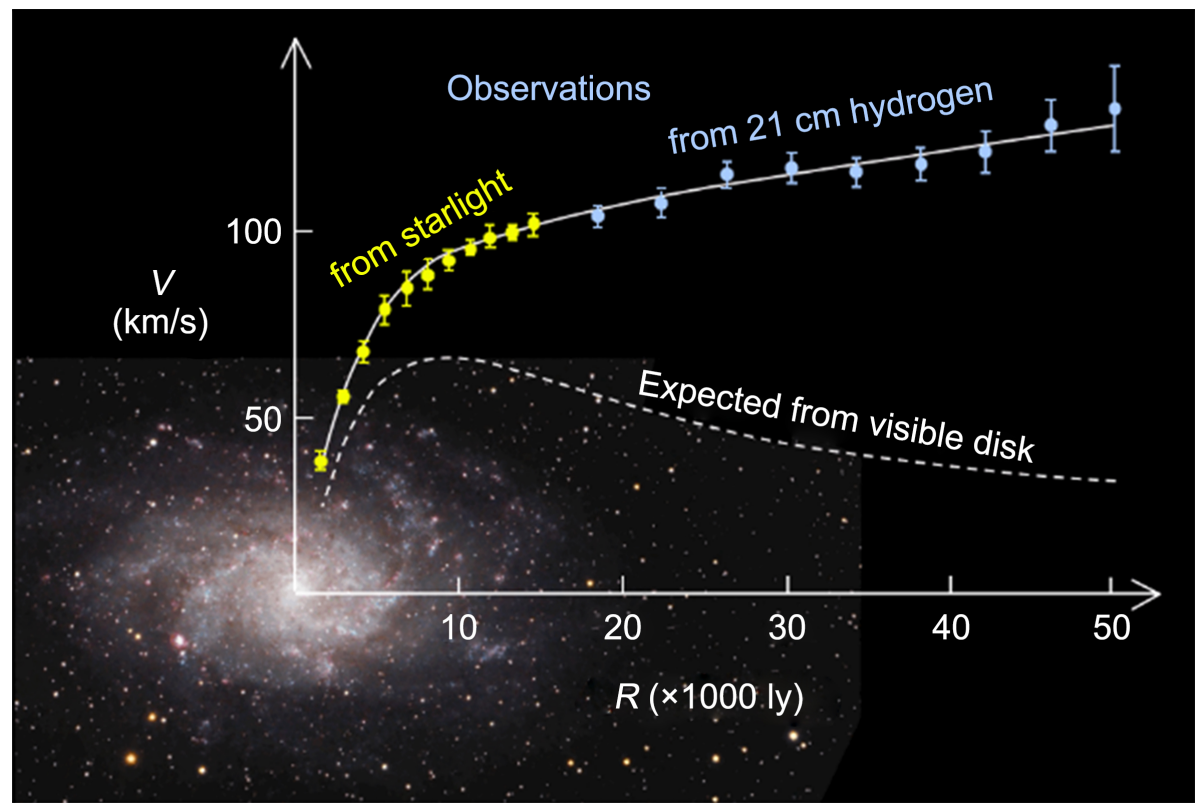

Figure 1. The rotation curve of galaxies measured by neutral hydrogen: the measured rotation velocity of the neutral cold gas is much larger than the Kepler velocity calculated by the total mass of visible glowing matters in the galaxy. This image is taken from the NASA website. 
Brian P. Schmidt, and Adam G. Riess for their discovery of the accelerating expansion of the universe. It is dark energy that causes the accelerating expansion of the universe. However, we do not know anything about its nature, except that it is the dominant component of the universe, accounting for about $70 \%$ of all the energy in the universe. Most of the rest is dark matter, which makes up about $25 \%$ of the energy of the universe. The common baryonic matter, on the contrary, makes up only about $5 \%$ of the universe, and most of it is ionized gas as shown in Figure 2.

According to the data observed by Planck Satellite in 2015, the age of the universe is $13.787 \pm 0.020$ Gyr, the Hubble's constant is $67.66 \pm 0.42 \mathrm{~km} \cdot \mathrm{s}^{-1} \cdot \mathrm{Mpc}^{-1}$, the dark energy density parameter is $0.6889 \pm$ 0.0056 , and the material density parameter is $0.3111 \pm 0.0056$ [2].

$10^{-34} \mathrm{~s}$ after the Big Bang, the temperature of the universe was about $10^{27} \mathrm{~K}$. At that moment, the Big Bang stopped and the sum of the material density parameter and the dark energy density parameter approached one. Two seconds after the universe formed, neutrons stopped forming. Three minutes after, the abundance of light elements was almost fixed. Sixty-five thousand years later, the main component of the universe changed from radiation to mass, and the structure of dark matter began to form. The microwave background radiation was nearly fixed 400,000 years later. About 10 to 100 million years later, stars began to form. Six billion years later, the expansion of the universe was accelerated by dark energy instead of slowing down.

\subsection{Status of Dark Matter Research}

It is indicated by the astronomical observations that dark matter is abundant in galaxies, galaxy clusters, and the universe. In the standard cosmological model $(\Lambda \mathrm{CDM})$, dark matter accounts for $85 \%$ of the mass of total matter in the whole universe. Unlike those easily observable morphologies of cosmic matter such as stars, dust, and gas, dark matter cannot be directly detected by electromagnetic means. Currently, astronomers measure the mass and mass distribution of dark matter by its gravitational effect. Although dark matter has been discovered for more than 100 years and it is abundant in the universe, still we do not know what it is.

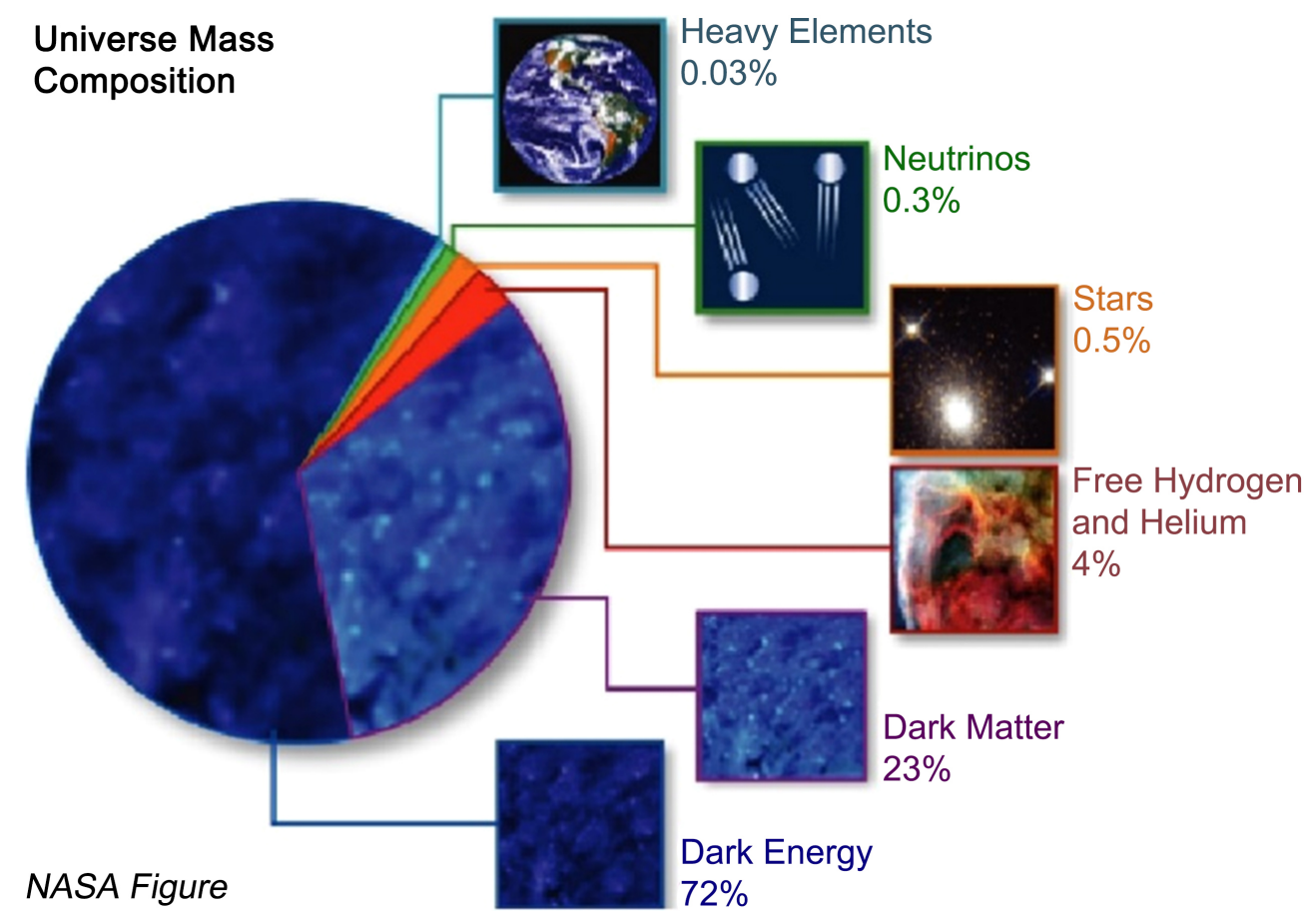

Figure 2. The major components and their proportions of the current universe. This image is taken from the NASA website. 
There are two types of candidates for dark matter. One is astrophysics candidates (compact objects without radiation), such as low mass star whose mass is less than the limit of hydrogen fusion $\left(<0.1 M_{\odot}\right)$, cold compact stars (white dwarfs, neutron stars), or stellar-mass black holes, etc. The other is particle physics candidates like weakly interacting massive particles, axions, and inert neutrinos, which do not participate in electromagnetic interactions. On December 17, 2015, China successfully launched the Dark Matter Particle Explorer "Wukong" into space. Wukong has been in orbit for five years, taking the dark matter detection of China to an unprecedented level [3].

Particle physics candidates for dark matter are difficult to form complex structures due to their limited interaction scope and exist in the gravitational field in a dispersed morphology. This is completely different from the compact morphology of the astrophysics candidates for dark matter. Modern cosmology considers that most cosmos matter is dark matter. It will provide a direction for the dark matter study by determining whether the dark matter is in a dispersed or compact morphology.

\subsection{MACHOs Experiment in the Milky Way Galaxy and the MACHOs Detection in the Universe}

The practical application of using microlensing light to detect the morphology of dark matter focuses on the study of dark halo in the Milky Way Galaxy. In 1986, Paczyński proposed to define the morphology of dark matter in the dark halo in the Milky Way Galaxy by the detection ratio of microlensing events for distinguishable stars in nearby galaxies. This type of microlensing light has a time scale of days and is easier to monitor. The optical thickness $(\tau)$ of the gravitational lens of dark halo in the Milky Way Galaxy is only about $10^{-6}$, which has a very low incidence of microlensing events, expecting that several events can be detected in a year by monitoring a large number of stars. Since 1990, numerous projects represented by the MACHO (Massive Astrophysical Compact Halo Object) experiment have conducted intensive field monitoring of compact stars in the Large Magellanic Cloud galaxy, the Small Magellanic Cloud galaxy, and the Galactic spheres. Several microlensing light events have been detected by each project, but no statistically significant results have been obtained.

In 1973, William H. Press and James E. Gunn proposed a method to measure the mass density of compact matter in the universe by gravitational lens effect and established the corresponding calculation and statistical model. The Press \& Gunn method uses high spatial resolution radio interference imaging surveys to detect multiple images of high-redshift celestial bodies formed by the gravitational lens effect of compact matter in the foreground stars and to estimate the mass density of compact matter in the universe. The method requires a large enough "lens" celestial body to form distinguishable multiple images. The distance between the multiple images of high-redshift celestial bodies is about $3 \times 10^{-6}\left(M / M_{\odot}\right)^{1 / 2}$ arcsecond. The resolution of sub-milliarcsecond can only detect compact matters with a mass of larger than $M_{\odot}^{4}$.

Compared with the direct detection of multiple images caused by gravitational lens effects, it is easier to detect the compact matter of stars by monitoring the high-redshift celestial body light in the intermediate matter passing through the sight caused by the microlensing effect. Analytical calculation and simulation have shown that the incidence of microlensing events in high-redshift objects is observable. If the dark matter in the universe is in the dispersed morphology, the incidence of microlensing events of high-redshift celestial bodies entirely randomly caused by stars is $1 \%$ at any moment. If the dark matter in the universe is compact, the incidence is as high as $10 \%$.

The morphology of dark matter in the universe can be defined by monitoring the high-redshift celestial bodies and counting the detection ratio of microlensing light events.

\section{THEORETICAL ANALYSIS}

\subsection{Physics of Microlensing Events}

Soon after Einstein proposed the General Theory of Relativity, he predicted that in a strong gravitational field light would deflect. The General Theory of Relativity speculates that space will be distorted by 
mass. In a gravitational field, light travels at a speed slower than the speed of light from the perspective of a distant observer, in which case gravity is the index that causes light to refract. According to Fermat's principle, thus, light bends in the direction of mass as it travels in an uneven gravitational field. In other words, light deflects in a strong gravitational field.

Later, a group of scientists led by Sir Arthur Eddington confirmed the gravitational lens phenomenon during a solar eclipse. Although Einstein believed that the gravitational lens was a phenomenon with a very low chance and could not be found in astronomical observation, it has become an important research method in astrophysics with the progress of technology.

To better understand the gravitational lens phenomenon and the lens equation, let us consider the following scenario. A beam of light from a distant source is approaching a particle. The shortest distance from the light to the particle is $b$, and the light bends $\alpha$ rads toward the particle. In this scenario, $b$ should be much larger than the Schwarzschild radius of the particle, which can be expressed by the formula:

$$
\frac{G M}{c^{2} b} \ll 1
$$

Meanwhile, the radius can significantly bend the light, that is, $b$ must be much shorter than the length involved in other scenarios. This is called the "thin lens approximation". Under the conditions of the thin lens approximation, we can obtain a simple expression for the bending angle of the light:

$$
\alpha=\frac{4 G M}{c^{2} b}=\frac{2 r_{s}}{b} v
$$

Once the expression for the bending angle of the light is obtained, consider the following three elements, i.e. point light source, particle, and observer forming a line, as shown in Figure 3.

In this scenario, from the above two equations, we can get:

$$
\frac{4 G M}{c^{2} D_{0 l} \theta_{E}} D_{l s}=\theta_{E} D_{o s}
$$

where $D_{l s}, D_{o l}, D_{o s}$ are distance between lens and source, distance between observer and lens, and distance between observer and source respectively.

And the expression of the Einstein angle can be deduced as:

$$
\theta_{E}=\left(\frac{4 G M}{c^{2}} \frac{D_{l s}}{D_{o l} D_{o s}}\right)^{1 / 2}
$$

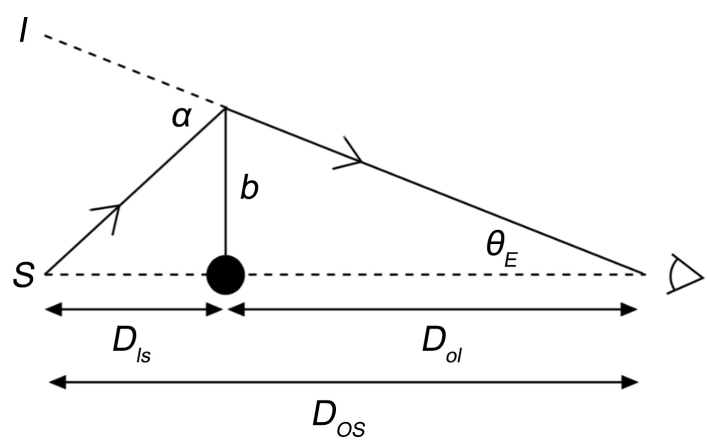

Figure 3. In this figure, $S$ stands for the point light source, the solid circle stands for the particle, and the "eye" stands for the observer. In this case, only the light emitted in a specific angle can reach the observer by the deflection effect from the particle, forming a ring in the observer's view, namely the Einstein ring. The angular radius is $\theta_{E}$ of which the projection on the lens plane is $r_{E}$ [4]. 
In most common cases, however, the point light source, particle, and observer are not in a line, as shown in Figure 4. The light source deviates from the particle a degree of $\beta$. This situation still belongs to the thin lens approximation, where all angles are minimal, and $b$ is much smaller than other lengths. Therefore, it can be concluded that:

$$
\begin{gathered}
D_{l s} \alpha+D_{o s} \beta=D_{o s} \theta \\
\beta=\theta-\frac{D_{l s}}{D_{o s}} \alpha=\theta-\frac{D_{l s}}{D_{o s}} \frac{4 G M}{c^{2} D_{o l} \theta}=\theta-\frac{\theta_{E}^{2}}{\theta} \\
\theta^{2}-\beta \theta-\theta_{E}^{2}=0
\end{gathered}
$$

Solve this quadratic equation with one variable and the angle formed by the virtual image and the lens-observer can be obtained, namely:

$$
\theta_{ \pm}=\frac{1}{2}\left[\beta \pm\left(\beta^{2}+4 \theta_{E}^{2}\right)^{1 / 2}\right]
$$

Next, we need to consider the magnification of the light source caused by microlensing, as shown in Figure 5. The light source not affected by the lens is located at the angle of $\beta$, and its radial angle is $\mathrm{d} \beta$. The light source affected by the lens is located at the angle of $\theta_{+}$and $\theta_{-}$, and the corresponding radial angle is $\mathrm{d} \theta_{+}$and $\mathrm{d} \theta_{-}$, respectively. The mirror of $\theta_{+}$is tangentially stretched to $\left(\theta_{+} / \beta\right)$ times and radially stretched to $\left(\mathrm{d} \theta_{+} / \mathrm{d} \beta\right)$ times.

Therefore, the solid angle ratio formed by the mirror and light source is $\theta_{ \pm} \mathrm{d} \theta_{ \pm} / \beta \mathrm{d} \beta$, and its relative change can be expressed as:

$$
\alpha_{ \pm}=\frac{\theta_{ \pm} \mathrm{d} \theta_{ \pm}}{\beta \mathrm{d} \beta}=\frac{\theta_{ \pm}}{2 \beta}\left[1 \pm \frac{\beta}{\left(\beta^{2}+4 \theta_{E}^{2}\right)^{1 / 2}}\right]
$$

In the microlensing event, the surface brightness of the target source image is conserved. The increase of the angle means the enhancement of the brightness, from which we can get the expression of the change of brightness:

$$
a_{t o t}=a_{+}+a_{-}=\frac{u^{2}+2}{u\left(u^{2}+4\right)^{1 / 2}}
$$

where $u \equiv \frac{\beta}{\theta_{E}}$ is the minimum collision distance of units in the Einstein ring.

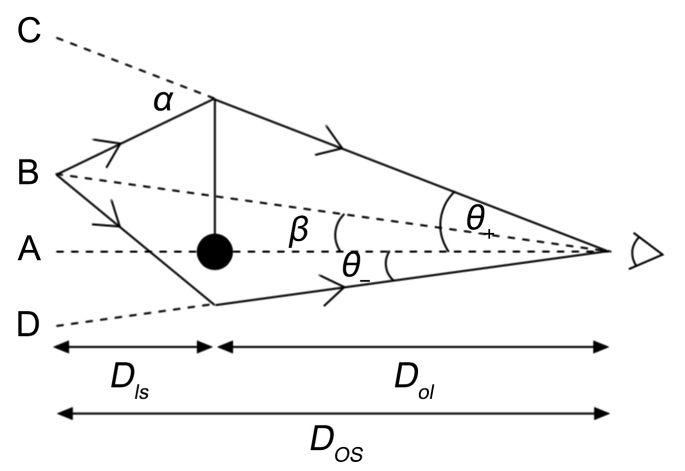

Figure 4. In this figure, B stands for the point light source, the solid circle is the particle, and the "eye" on the far right is the observer. In this case, the point light source, particle, and observer are not in a line, which is different from the line situation. The chance of forming a perfect line in the universe is pretty low, so this situation is more common [4]. 
In the case of a single lens, the model in the lens plane can be generalized as shown in Figure 6.

Accordingly, we can get the equation of $u$ :

$$
u(t) \equiv \frac{\beta(t)}{\theta_{E}}=\left[u_{0}^{2}+\frac{\left(t-t_{0}\right)^{2}}{\tau^{2}}\right]^{1 / 2}
$$

where $u_{0} \equiv \frac{\beta_{0}}{\theta_{E}}, \tau \equiv \frac{\theta_{E} D_{l o}}{v}$. Since the relation between the enhancement ratio of brightness and $\mathrm{u}$ has been obtained, the light curve of the single lens can be obtained directly.

We used MulensModel software package to construct the light curve of the microlensing event of a single star and discussed the change of light curve when different parameters were set shown in Table 1. The MulensModel package is freely available on GitHub (https://github.com/rpoleski/MulensModel).

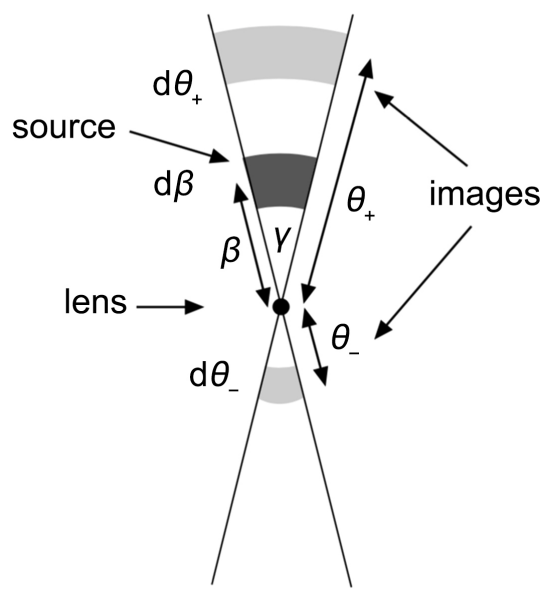

Figure 5. In this image, the light source not affected by the lens is located at the angle of $\beta$, and its radial angle is $\mathrm{d} \beta$. The light source affected by the lens is located at the angle of $\theta_{+}$and $\theta_{-}$, and the corresponding radial angle is $\mathrm{d} \theta_{+}$and $\mathrm{d} \theta_{-}$, respectively [4].

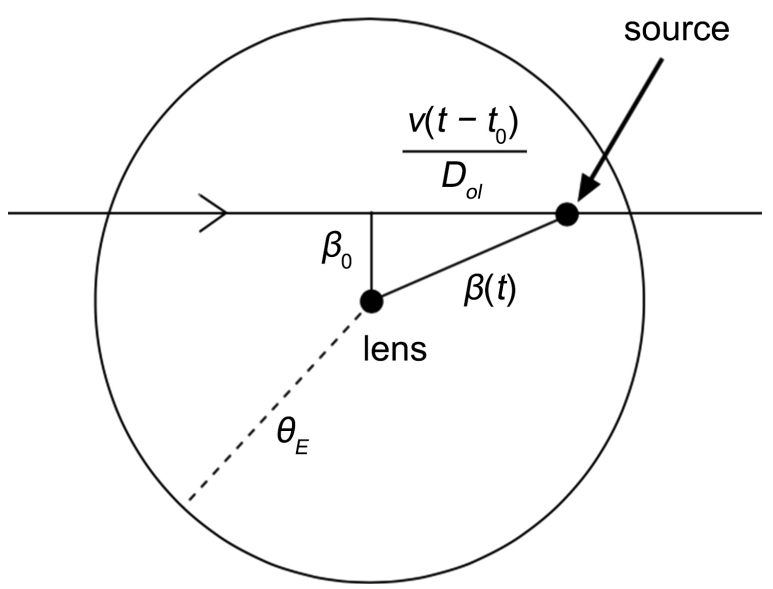

Figure 6. In this figure, the point marked with "lens" stands for the particle, the "source" is the point light source. The direction of the observer's sight is perpendicular to the paper, and the straight line marked with an arrow is the movement track of the point light source. $\beta_{0}$ is the minimum projection angle of the point light source to the particle center. $\beta(t)$ is the dynamic projection angle as a function of time. 
Table 1. Light curves of microlensing events with various parameters, illuminating the effects of crossing time and impact parameter.

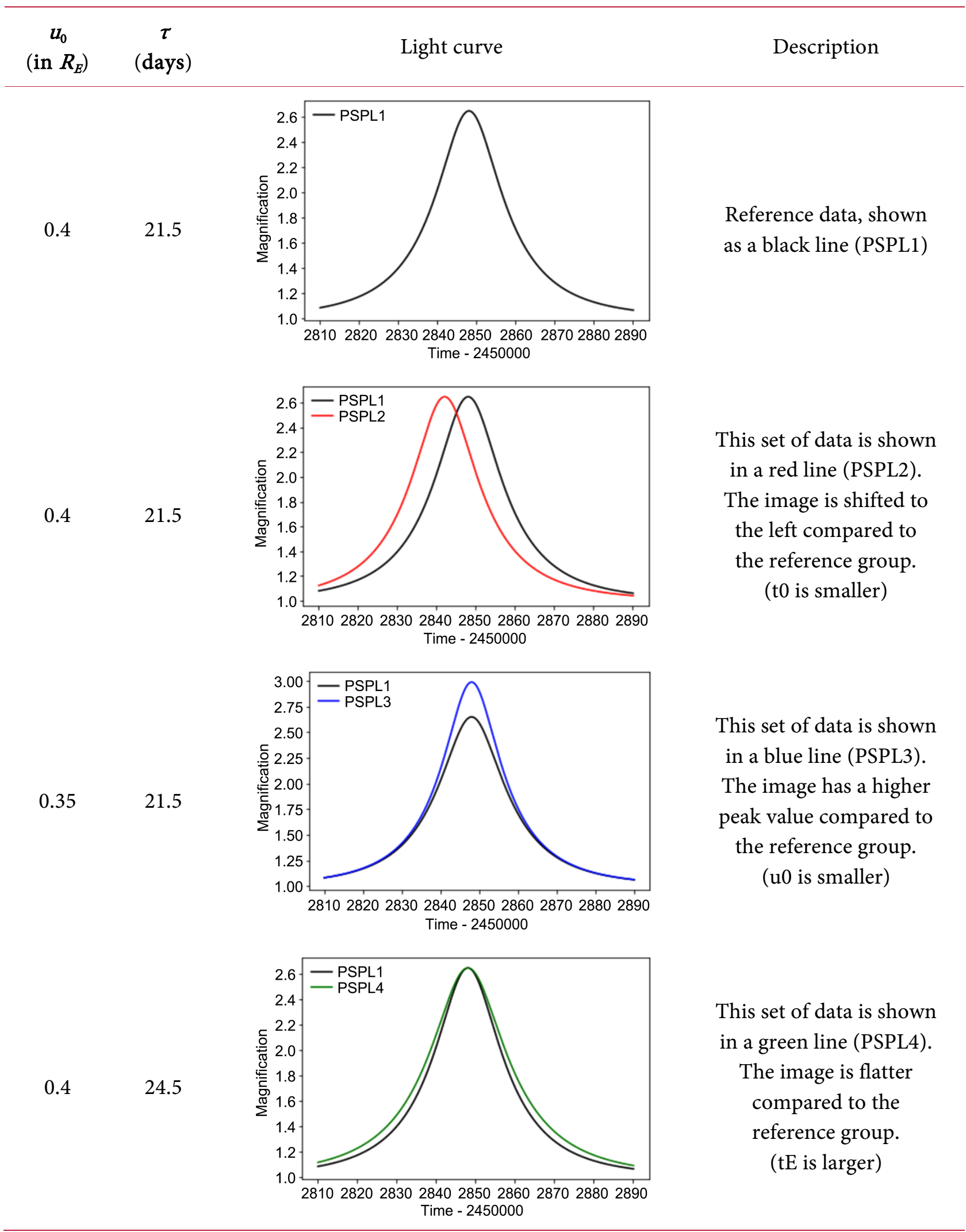




\subsection{Cosmological Distance Calculation}

From the perspective of the principle of physics, microlensing is very similar to geometrical optics. Except for the lens nature determines the imaging effect, the distance (including the distance from the observer to the lens, that from the observer to the light source, and that from the lens to the light source) also directly affects the imaging effect. Below we discuss the calculation of cosmological distance and apply it to microlensing events.

Cosmological distance differs from the definition of space in Euclidean geometry. There are various definitions of distance, or distance itself is different as the definition of measurement changes. In Euclidean geometry, however, these distances are the same, so they are not distinguished. Distance commonly discussed in cosmology includes co-moving distance, luminosity distance, and angular diameter distance (Hogg 2000). Microlensing is a geometric phenomenon that angular diameter distance applies.

The first is co-moving distance. The co-moving distance between point $\mathrm{A}$ and point $\mathrm{B}$ is defined as the definite integral of the speed of light with respect to time. The lower limit and upper limit of the integral is the moment of light departs from $\mathrm{A}, t_{0}$, and the moment of light arrives $\mathrm{B}, t_{1}$, respectively. It can be seen that the definition of co-moving distance is very similar to the ordinary Euclidean distance. But the difference is that co-moving distance needs to consider the expansion of the universe. The universe is expanding, which makes the universe at this moment bigger than the universe at the last moment. Since light has traveled more distances within the same time, the speed of light at this moment can be equivalently regarded greater than that at the previous moment. Thus, the co-moving distance needs to be defined by the integral. The co-moving distance is related to the redshift value. The greater the redshift value is, the greater the co-moving distance will be.

The luminosity distance is the distance that links the intrinsic luminosity of the luminous object and the observed luminosity. In the case of the isotropic radiation energy, the luminosity distance can be simply expressed as $D \equiv \sqrt{\frac{L}{4 \pi f}}$, where $L$ stands for celestial intrinsic luminosity, $f$ stands for the observed luminosity flux.

Next, we discuss the angular diameter distance we need to use. We know that the radian is defined as the ratio of arc length to the radius, so the ratio of arc length to radian is the radius. In astronomical observation, we can only measure the viewing angle of a star, that is, the maximum angle between our two sightlines tangent to the star. Therefore, the angular diameter distance is defined as the ratio of an object's true diameter to its viewing angle. There is a characteristic of angular diameter distance. When the co-moving distance between the object and us remains unchanged, the angular diameter distance first increases with the redshift value and reaches its maximum value when the redshift value is about 1.5 , then decreases with the redshift value.

Using the astropy.cosmology software package, we calculated the angular diameter distance as a function of redshift value, as shown in Figure 7, in which there is a maximum value of the angular diameter distance. The relationship between the angular diameter distance and co-moving distance is $D A=\chi /(1+z)$, where $\chi$ is co-moving distance and $z$ is the redshift value. When $z$ increases, the numerator and denominator both increase, and when $z$ is large enough, the denominator dominates, resulting in that the angular diameter distance decreases with the redshift value.

Note that the calculation of the cosmological distance is directly related to the selection of the cosmological model. At present, the cosmological model parameters measured in different experiments are still different. In general studies of cosmological problems, it is necessary to explain the specific cosmological model under which the research is carried out.

\subsection{Background Light Source of Apparent Superluminal Motion}

From the above, we can see that distances involved in cosmological objects are very large, and the measuring unit is $\mathrm{Mpc}$, where $1 \mathrm{Mpc} \equiv 3.0856776 \times 10^{19} \mathrm{~km}$. At this distance, it is very slow to change the 


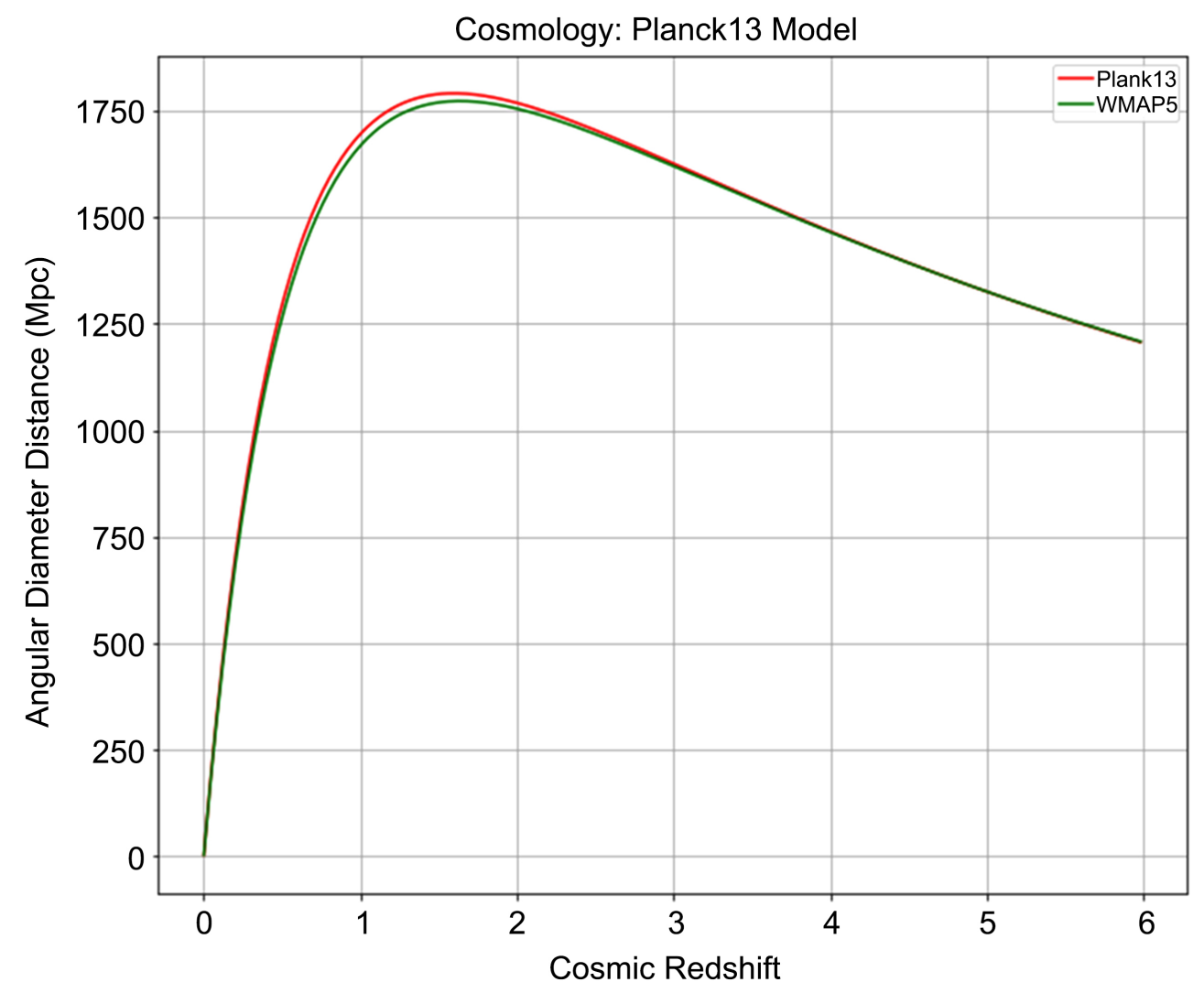

Figure 7. The angular diameter distance calculated under the Plank13 and WMAP5 cosmological models.

geometry of the objects by moving. This challenge will be discussed in more detail in the next section. To deal with this challenge, we need to observe objects that move very fast to detect cosmic microlensing events.

Quasars, as the name suggests, are special celestial bodies that resemble stars. The most important feature of quasars is that they are so bright that they can be seen from distances of up to 10 billion light-years. Quasars are difficult to distinguish from common stars by general optical means. They can only be distinguished by their radio radiation. Quasars shoot jets in both directions perpendicular to the accretion disk. The observation shows that the speed of the jet is faster than the speed of light, which actually is an apparent superluminal phenomenon. Actually, the speed of the jet is not faster than the speed of light, but only close to it. This apparent superluminal phenomenon occurs at the angle between the observer's sightline and the jet. When the sightline is perpendicular to the jet, the observed speed is the real speed of the jet. As the angle becomes smaller, the difference between the observed speed and the real speed gets greater.

As shown in Figure 8, $O$ point is the observer, $A B$ segment is the moving direction of the jet source, considering the source is in $A$ at the $t_{1}$ moment and in $B$ at $t_{2}$ moment. The observer can only see the projection movement of the jet in the sky, namely the $C B$ segment. The angle between the sightlines of $O A$ and $A B$ is $\theta$. Meanwhile, the observed movement of the jet source in the viewing angle of the observer is very small, namely $\phi \ll 1$.

Then, $A B=v \delta t, A C=v \delta t \cos \theta, B C=v \delta t \sin \theta$.

Considering the time for the observer to observe the radiation of the light source at different positions, marked with a prime, then:

$$
t_{2}-t_{1}=\delta t
$$




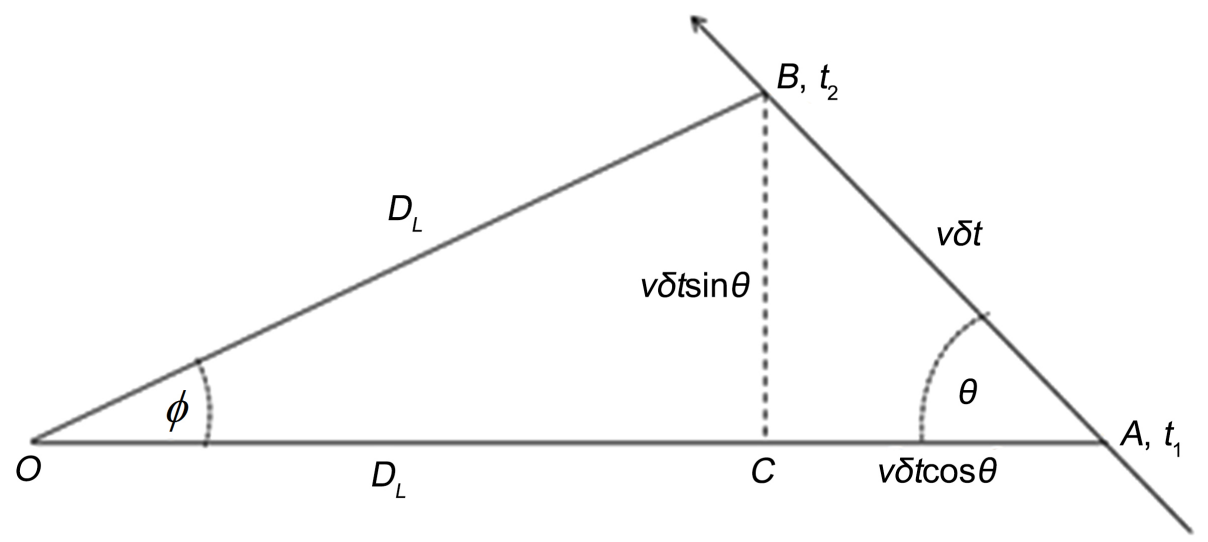

Figure 8. Schematic of apparent superluminal motion (Peterson, 1997).

$$
\begin{gathered}
t_{1}^{\prime}=t_{1}+\left(D_{L}+v \delta t \cos \theta\right) / c \\
t_{2}^{\prime}=t_{2}+D_{L} / c \\
\delta t^{\prime}=t_{2}^{\prime}-t_{1}^{\prime}=t_{1}-t_{2}-v \delta t \frac{\cos \theta}{c}=\delta t-v \delta t \frac{\cos \theta}{c}
\end{gathered}
$$

When discussing the relativistic problem, we generally use the normalized factor for the speed of light, namely $\beta=v / c$. So, the above equation can be described as $\delta t^{\prime}=\delta t(1-\beta \cos \theta)$. In contrast, $\delta t=\delta t^{\prime} /(1-\beta \cos \theta)$.

$$
B C=D_{L} \sin \phi \approx \phi D_{L}=v \delta t \sin \theta=\frac{v \sin \theta \delta t^{\prime}}{1-\beta \cos \theta}
$$

The projected velocity of motion from the observer's view is

$$
\begin{gathered}
V_{o b s}=\frac{B C}{\delta t^{\prime}}=\frac{v \sin \theta}{1-\beta \cos \theta} \\
\beta_{o b s}=\frac{\beta \sin \theta}{1-\beta \cos \theta}
\end{gathered}
$$

By analyzing the function, we can find that when the speed of the jet is close to the speed of light, namely $\beta \approx 1$, and when the angle between the jet and the observer's sightline is very small, namely $\theta \ll 1$, the observed projected speed of the jet by the observer exceeds the speed of light, i.e. it is an apparent superluminal motion.

\subsection{Cosmic Microlensing Events of Compact Dark Matter}

The cosmological distance calculation presented in Section 2.2 shows that the distances are very large when deep-space (high-redshift) objects are involved. The Einstein ring of the microlensing event of a celestial body is also very large. Besides, considering the average relative velocity between galaxies in the universe which is about $1000 \mathrm{~km} / \mathrm{s}$, and taking it as the characteristic velocity of relative motion between celestial bodies in the universe. Then, the time scale of the entire microlensing light event is very long, which can reach decades or even hundreds of years that cannot be observed. Fortunately, there exists a class of celestial bodies in the universe with apparent superluminal motion. Some quasars have strong apparent superluminal jets, which provide us an opportunity to effectively detect intermediate lens objects through microlensing events. Then, by calculating the time of the formation of microlensing events of compact dark matter objects with different masses in the case of different apparent superluminal celestial light sources, we evaluate the effective detection range of the redshift and mass of the compact dark matter in 
the reasonable observation time.

\subsubsection{Cosmic Microlensing Model}

When reviewing our discussion of microlensing in or near the Milky Way galaxy, we used to project the Einstein ring and the relative motion of the observer, lens object, and celestial light source onto the lens plane [5]. In the lens plane, the Einstein ring is:

$$
R_{E}=\theta_{E} D_{l}=\left(\frac{4 G M}{c^{2}} \frac{D_{l s}}{D_{l} D_{s}}\right)^{1 / 2} D_{l}=\left(\frac{4 G M}{c^{2}} \frac{D_{l s} D_{l}}{D_{s}}\right)^{1 / 2}
$$

However, in the discussion of microlensing time at a cosmological distance, it is customary to project the Einstein ring and the relative motion of the observer, the lens object, and the celestial light source to the light source plane. In the light source plane, the Einstein ring is:

$$
R_{E}=\theta_{E} D_{s}=\left(\frac{4 G M}{c^{2}} \frac{D_{l s}}{D_{l} D_{s}}\right)^{1 / 2} D_{s}=\left(\frac{4 G M}{c^{2}} \frac{D_{l s} D_{s}}{D_{l}}\right)^{1 / 2}
$$

The distances in the above equations are all angular diameter distances.

It is beneficial to take the light source plane as the basic plane in the calculation of cosmic microlensing because it becomes simpler to calculate the change in the velocity of the light source measured by the observer. Now we discuss how to calculate the velocities of the observer, lens, and light source to the relative motions in the plane of the light source measured by the observer.

In cosmology, it is not as simple to deal with relative motion at different redshifts as it is in Newtonian mechanics. General relativistic effects such as cosmological redshifts need to be considered.

Let the velocity of lateral movement of the light source be $v_{s}=\mathrm{d} s_{s} / \mathrm{d} t_{s}$. All measurements are made in the stationary reference frame of the light source, namely in the plane of the light source. Within the plane of the lens, the lateral movement speed of the lens is $v_{l}=\mathrm{d} s_{l} / \mathrm{d} t_{l}$. In the stationary reference frame of the observer, the observer's speed is $v_{o}=\mathrm{d} s_{o} / \mathrm{d} t_{o}$. then:

Project the distances measured in the observer plane and the lens plane to the light source plane,

$$
\begin{gathered}
\mathrm{d} s_{l}^{\prime}=-\frac{D_{s}}{D_{l}} \mathrm{~d} s_{l} \\
\mathrm{~d} s_{o}^{\prime}=-\frac{D_{l s}}{\left(1+z_{l}\right) D_{l}} \mathrm{~d} s_{o}
\end{gathered}
$$

Project the time measured in the observer plane and the lens plane onto the light source plane, then:

$$
\begin{aligned}
\mathrm{d} t_{l}^{\prime} & =\frac{1+z_{l}}{1+z_{s}} \mathrm{~d} t_{l} \\
\mathrm{~d} t_{o}^{\prime} & =\frac{1}{1+z_{s}} \mathrm{~d} t_{o}
\end{aligned}
$$

Thus, the relative motion velocity of the observer, lens, and light source measured in the plane of the light source is:

$$
v^{\prime}=v_{s}-\frac{1+z_{s}}{1+z_{l}} \frac{D_{s}}{D_{l}} v_{l}+\frac{1+z_{s}}{1+z_{l}} \frac{D_{l s}}{D_{l}} v_{o}
$$

When the observer makes measurements, the time redshift shall be considered, then:

$$
V=\frac{1}{1+z_{s}} v_{s}-\frac{1}{1+z_{l}} \frac{D_{s}}{D_{l}} v_{l}+\frac{1}{1+z_{l}} \frac{D_{l s}}{D_{l}} v_{o}
$$


Further, considering the apparent superluminal motion of the light source, the velocity measured by the observer in the above equation is dominated by the motion of the light source, which can be simplified as follows:

$$
V=\frac{1}{1+z_{s}} v_{s}
$$

Note that the apparent superluminal motion is a special relativistic effect. The above discussion is a revision of the observer's measured velocity considering the general relativistic effect in cosmology, which is not a repeated consideration of the relativistic effect.

\subsubsection{Analysis of the Crossing Time of the Cosmic Microlensing Events}

In the above section, we derive the scale of the Einstein ring in the light source plane and the apparent superluminal motion measured by the observer. The crossing time of the microlensing events is their quotient, i.e. $\tau=R_{E} / V$. Below, we calculate the crossing time of the typical cosmic microlensing event to analyze the observability of these events.

Generally, the redshift of the light source is between 0.1 and 3, while the redshift of the lens is $1 / 2$ of the redshift of the light source. The speed of light source is set as between 0.5 and 7 times the speed of light. The mass of the lens takes $1 M_{\odot}$ and $10 M_{\odot}$.

The crossing time calculated in the selected parameter range is basically within one year. Conventional observatories at medium latitudes only have an observable window of 6 months for celestial bodies in a year, so the light curve of microlensing events with a crossing time over 6 months cannot be completely covered. Strictly speaking, events with a crossing time over 6 months are considered unobservable. For events with a crossing time of fewer than 6 months, they can be fully observed in one observing season. But it depends on the relationship between the time of the event and the observable window of the target object.

More conservatively, we consider the events with a crossing time of less than one month as observable events. If the above requirement is adopted, the compact dark matter object whose light source velocity is more than 0.5 times the speed of light and whose lens mass is less than 1 solar mass can be well observed by using the microlensing event. For compact dark matter objects with a mass of 10 solar masses, the speed of the light source needs to be larger than twice the speed of light.

The relationship between the crossing time and the mass and speed of the object is very direct, that is $\tau \propto \sqrt{M}$ and $\tau \propto V^{-1}$, respectively. Therefore, the crossing time of other situations can be determined as shown in Figure 9. If the mass of a compact dark matter object is greater than 100 solar masses, it is not observable when taking one month as the detection threshold.

\section{DISCUSSION}

\subsection{Estimation of Detection Probability}

In the previous chapter, we have calculated in detail the basic principle and feasibility of the microlensing event of the apparent superluminal source. In this section, we focus on the detection probability, i.e. the relationship between the morphology of dark matter and the detection probability of cosmic microlensing events.

To calculate the detection probability, we should first calculate the volume density of the dense dark matter in the universe. Note that the volume of the universe varies, so the discussion of volume density in cosmology must consider the volume variation of the universe. Here, we need to use the cosmological concept of coactive volume. It is the volume defined in the case that the volume density of a celestial body does not change with the evolution of the universe (i.e. it is equal at different redshifts) (Hogg, 2000).

Without the limitation of the cosmological model, we can put the coactive volume at the solid angle of $\mathrm{d} \Omega$ and the redshift intervals of $\mathrm{d} z$ as: 


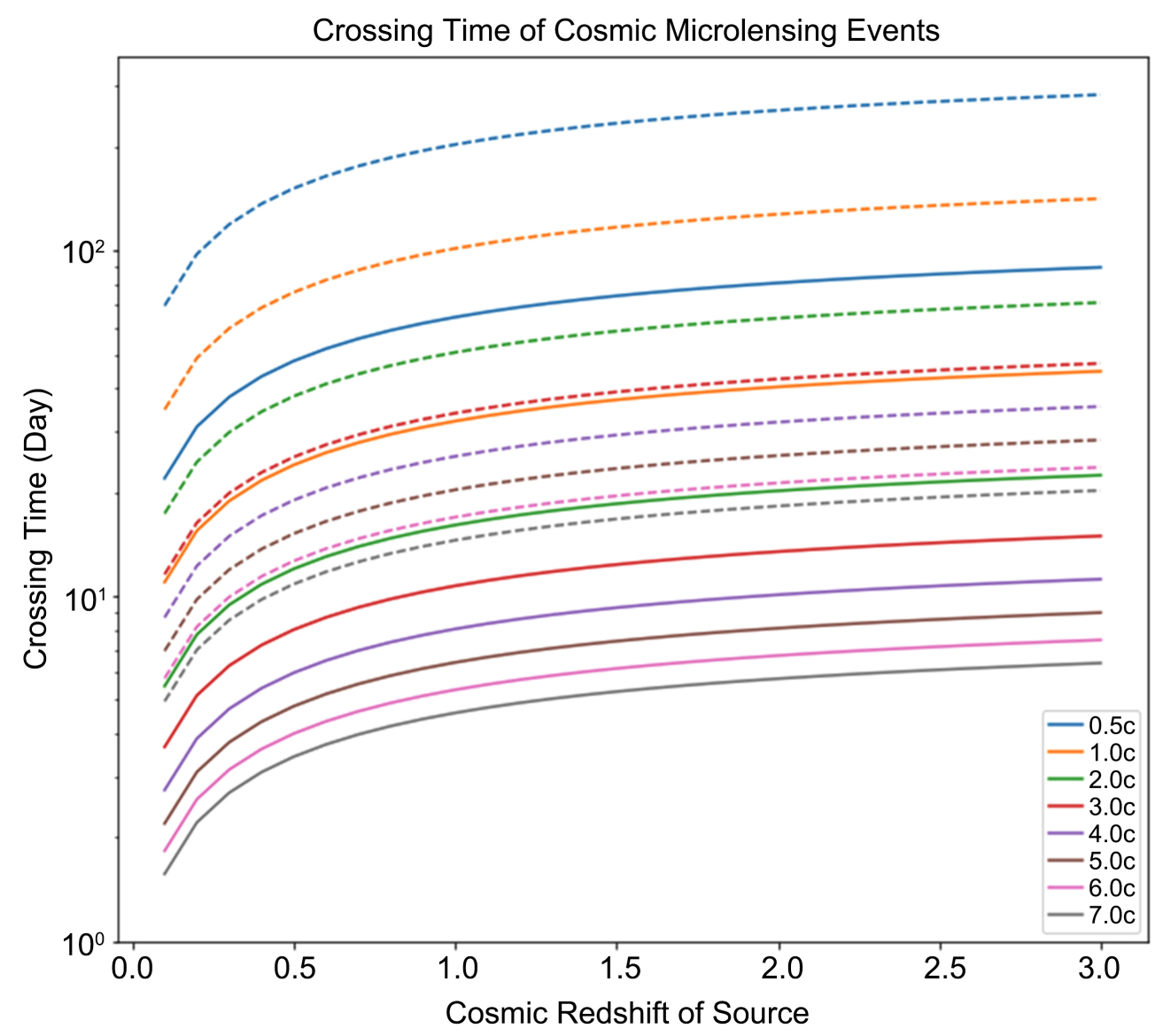

Figure 9. The crossing time of cosmic microlensing events. The redshift of the light source is 0.1 to 3 , and the redshift of the lens is $1 / 2$ of the redshift of the light source. The speed of the light source is 0.5 to 7 times the speed of light (curves in different colors). The mass of the lens is $1 M_{\odot}$ (solid line) and $10 M_{\odot}$ (dashed line). The unit of crossing time is days.

$$
\mathrm{d} V_{c}=\frac{c}{H_{0}} \frac{(1+z)^{2} D_{L}}{E(z)} \mathrm{d} \Omega \mathrm{d} z
$$

where

$$
E(z)=\sqrt{(1+z)^{4} \Omega_{r}+(1+z)^{3} \Omega_{m}+(1+z)^{2} \Omega_{k}+\Omega_{\Lambda}}
$$

In the current cosmological model (ignore the contribution brought by the radiation and curvature of the composition of the universe introduced in Section 1.2), $\Omega_{r}=0, \Omega_{k}=0$. Then, the coactive volume can be put as:

$$
\mathrm{d} V_{c}=\frac{c}{H_{0}} \frac{(1+z)^{2} D_{L}^{2}}{\sqrt{(1+z)^{3} \Omega_{m}+\Omega_{\Lambda}}} \mathrm{d} \Omega \mathrm{d} z
$$

If the compact dark matter in the universe has the same mass, then we can get the volume density in the current universe, which can be generalized to the whole universe through the coactive volume.

$$
n=f \Omega_{M} \rho_{c} / M
$$


where $f$ is the ratio of the compact dark matter in the universe, $\Omega_{M}$ is the ratio of matter in the current universe, $M$ is the given mass of the compact dark matter, $\rho_{c}=\frac{3 H_{0}^{2}}{8 \pi G}=8.6 \times 10^{-27} \mathrm{~kg} \cdot \mathrm{m}^{-3}$ is the critical density of the current universe.

So, if the density of lens in the universe is not high, we can ignore the intersection of the Einstein rings of the front lens and the behind lens in the sightline. In this case, it is easy to get the incidence of microlensing events:

$$
\tau\left(z_{s}\right)=\frac{1}{4 \pi} \int n \mathrm{~d} V\left(z_{l}\right) \pi \theta_{E}^{2}
$$

Further, substitute the expressions of the coactive volume and the Einstein ring, we can get:

$$
\tau\left(z_{s}\right)=f \frac{3 \Omega_{M} H_{0}}{2 c} \int_{0}^{z_{s}} \frac{(1+z)^{2}}{\sqrt{(1+z)^{3} \Omega_{M}+\Omega_{\Lambda}}} \frac{D_{l s} D_{o l}}{D_{o s}} \mathrm{~d} z
$$

The expression is the probability of a cosmic microlensing event along any sightline. The only key parameter in this expression is $f$, which is the ratio of compact matter in the whole matter. In our current understanding of the universe, most matter in the universe is dark matter. Therefore, it is reliable to regard $\mathrm{f}$ as the ratio of compact dark matter in the universe.

In the actual observation, a sample of the light source observed in the random direction has a sample size of $N$. The number of observed microlensing events is $m$. Then $m / N$ is compared with the above expression, which can get the ratio of compact dark matter in the universe. The above expression is graphed using numerical calculations (Figure 10), which can be conveniently compared with the actual observations.

\subsection{The Scan Speed of Apparent Superluminal Source over the Dark Matter in the Universe through a Microlensing Effect}

The concept of "apparent superluminal microlensing" was first used to explain the rapid light changes of quasars in the visible and radio wavelengths. The basic idea is that the sightline toward a bright spot (or hotspot) in the relativistic jets of the quasar can sweep across the distance of any galaxy at a visual transverse velocity (perhaps super velocity of light). In gravitational lensing, these bright spots can cause rapid light changes, which are measured in hours in visible wavelengths and days in radio wavelengths.

Apparent superluminal microlensing can significantly increase the detection probability of cosmic microlensing events within a specific monitoring period, especially in the case of more generally ordinary optical depths. Next, we calculate the velocity of the apparent superluminal light source scans the volume of the universe to deduce the gain of the probability of cosmic microlensing events [6].

To observe strong microlensing events, the point light source must be in the radius of Einstein, $R_{E}$. When a particle with a mass of $m$ is regarded as a microlensing, its radius of Einstein $R_{E}$ is as follows:

$$
R_{E} \approx\left(\frac{4 G m D}{c^{2}}\right)^{1 / 2} \cong 10^{-2} \mathrm{pc}\left(\frac{D}{500 \mathrm{Mpc}}\right)^{1 / 2}\left(\frac{m}{M_{\odot}}\right)^{1 / 2}
$$

where $D=D_{o l} D_{l s} / D_{o s}$, in which the distance is the angular diameter distance of the lens in the universe, the lens to the light source, and the light source, respectively.

In the foreground galaxy, the number of micro-gravitational lenses that can exert a strong influence on the stationary light source is:

$$
N_{*} \cong \frac{\pi r_{E}^{2} \Sigma(r)}{m}=\frac{\Sigma(r)}{\Sigma_{\text {crit }}}=\kappa(r)
$$




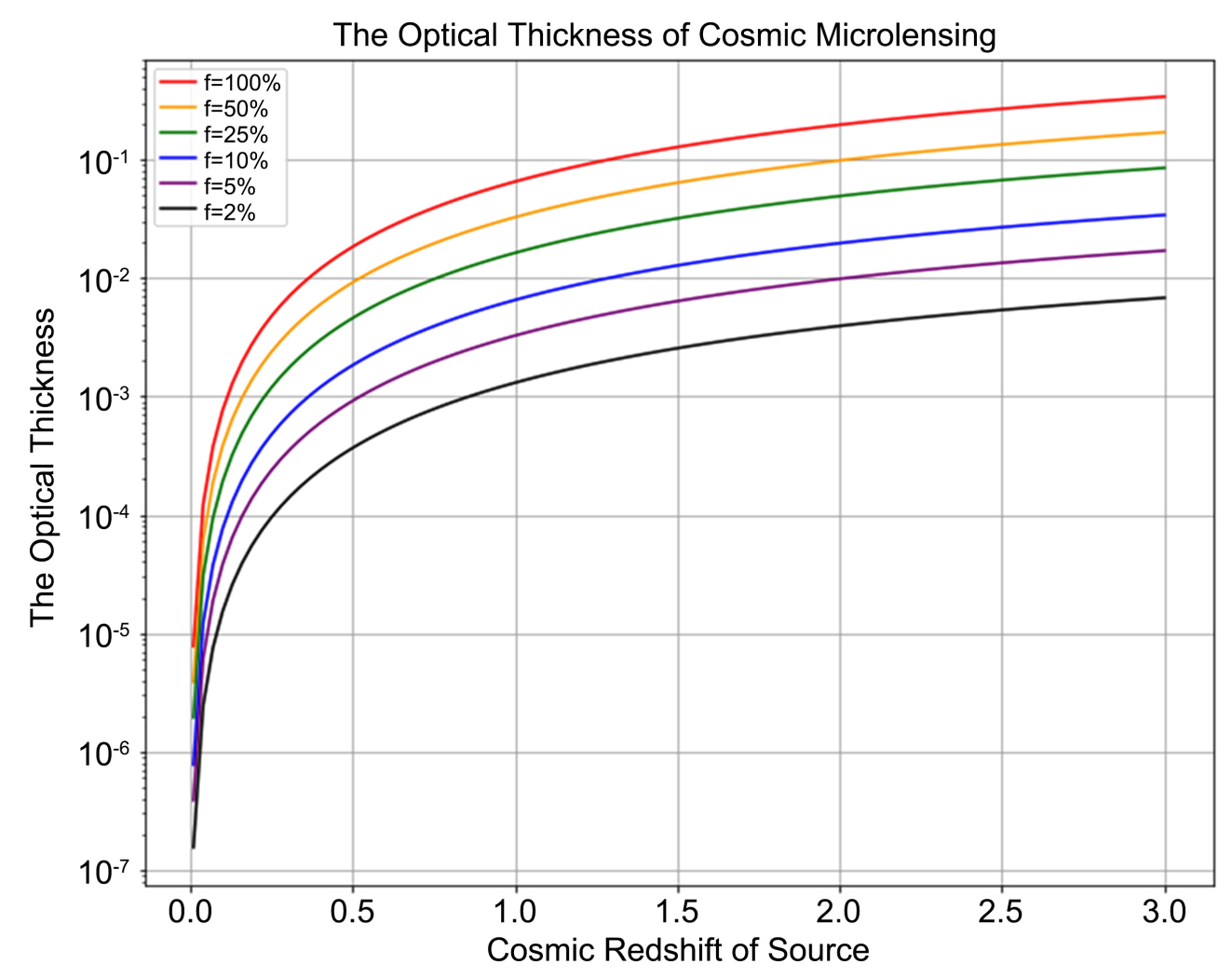

Figure 10. The relationship between the detection ratio of microlensing events and the ratio of compact dark matter in the universe was observed under light sources with different redshifts. A sample of the light source is observed in the random direction with a sample size of $N$. The number of the observed microlensing events is $\mathrm{m}$. Then, $\mathrm{m} / \mathrm{N}$ is compared with the figure above, which can obtain the ratio of compact dark matter in the universe.

In the above equation, $\Sigma(r)$ is the mass density of the micro-gravitational lens projected on the $r$ surface in the lensing galaxy; $\Sigma_{c r i t}=\frac{c^{2}}{4 \pi G D}$ is the critical surface density of the micro-gravitational lens. If the surface density of the micro-gravitational lens in a lensing galaxy equals the critical density, then space is filled with Einstein rings one after the one without intersection.

According to the existing knowledge, the bright spot of the relativistic jet of quasar moves at the speed of superlight visually, which is also much faster than the usual microlensing events. Correspondingly, we can get the idea that the number of microlensing events scanned by the sightline towards the bright spot of the relativistic jet of the quasar will be much larger than that scanned by other light sources. Therefore, within a given time $t$, the number of micro-gravitational lenses that will cause strong microlensing events to occur in the apparent superluminal jet is:

$$
N \approx\left(\pi r_{E}^{2}+\left[\frac{2 r_{E} \gamma c t}{1+z_{s}}\right] \frac{D_{o l}}{D_{o s}}\right) \frac{\Sigma(r)}{m}=\kappa(r)\left[1+\frac{t}{\tau}\right]
$$

where

$$
\tau=\frac{\pi}{2}\left[\frac{r_{E}\left(1+z_{s}\right)}{\gamma c}\right] \frac{D_{o s}}{D_{o l}} \approx 2\left(1+z_{s}\right)\left(\frac{\gamma}{10}\right)^{-1}\left(\frac{m}{10^{-3} M_{\odot}}\right)^{1 / 2}\left(\frac{\bar{D}}{1000 \mathrm{Mpc}}\right)^{1 / 2}
$$


is the crossing time of apparent superluminal microlensing event, in which $\bar{D}=\frac{D_{l s} D_{o s}}{D_{o l}}$. It is apparently that the probability of observing a microlensing event significantly increases $\eta=\kappa(t / \tau)$ times when continuously observing a light source within the time $t$.

\section{CONCLUSIONS AND PROSPECTS}

In 1998, Abraham Loeb and Rosalba Perna proposed to measure the density of compact matter in the universe by observing the microlensing events of the afterglow of gamma-ray explosions. The velocity of the gamma-ray burst afterglow ring is extremely relativistic and exhibits the apparent superluminal motion. This greatly reduces the crossing time of cosmic microlensing events to the scale of days. Meanwhile, since the speed of the background light source is fast, it greatly increases the volume of the universe scanned by the sightline within a certain time and enhances the ratio of event detection to an extent that every gamma-ray burst afterglow creates a microlensing event with a certain intensity [7]. The flaw of monitoring gamma-ray burst afterglow is that gamma-ray burst is a transient celestial body, which has limited observable targets abundant and a strict requirement of the observing time. At present, there is no conclusion on the morphology of dark matter in the universe obtained by gamma-ray burst afterglow monitoring.

The extreme relativistic jets of quasars are another high redshift background source that can be used for detecting cosmic microlensing events. Some of the observed low and medium frequency radio rapid light changes in quasars can be explained by the microlensing effect of the extreme relativistic jets [6].

According to Wyithe \& Turner's estimation of the detection ratio of microlensing events in the gamma-ray burst afterglow, the detection ratio of microlensing events in a single quasar is predicted to be 1 case per year if all the cosmic dark matter is compact [8]. If 100 quasars are monitored with telescopes and all dark matter in the universe is in compact morphologies, the number of events expected to be detected in a single observation season is about 30. In theory, the definition of dark matter morphology will reach the confidence level of $5 \sigma$.

The theoretical calculation and experimental design seem to be relatively perfect, but in fact, it is still very difficult to detect the microlensing light change of the quasar jets. The difficulty lies in that the behavior of the intrinsic light changes in quasars is complex (compared with the light curve of a gamma-ray burst that can be described by simple power-law). Therefore, it is necessary to design the observation strategy and data processing method to extract the microlensing event signal from the light curve.

The intrinsic light changes in quasars can be considered as random changes along with explosions. The random components in the quasar light curve are similar to red noise and can be easily distinguished from the light change of microlensing. There also exist some differences in the characteristics of the burst light changes and the microlensing light changes. The amplitude and contour of the latter are independent of the observed band, and the contour has symmetry, which can be selected in the multi-band continuous sampling light curves. Vedantham et al. detected multiple cosmic microlensing events of quasar jets using multi-band radio observations [9].

The method discussed in this paper to define the morphology of dark matter in the universe by using the statistical analysis of microlensing events of apparent superluminal celestial bodies is relatively perfect in theory. Its application to the observation requires the cooperation of multi-band equipment to carry out long-term monitoring.

\section{CONFLICTS OF INTEREST}

The authors declare no conflicts of interest regarding the publication of this paper.

\section{REFERENCES}

1. de Swart, J.G., Bertone, G. and van Dongen, J. (2017) How Dark Matter Came to Matter. Nature Astronomy, 1, 
59. https://doi.org/10.1038/s41550-017-0059

2. Planck Collaboration (2016) Planck 2015 Results. XIII. Cosmological Parameters. A\&A, 594, 13. https://doi.org/10.1051/0004-6361/201525830

3. DAMPE Collaboration (2017) Direct Detection of a Break in the Teraelectronvolt Cosmic-Ray Spectrum of Electrons and Positrons. Nature, 552, 63-66. https://doi.org/10.1038/nature24475

4. Maoz, D. (2007) Astrophysics in a Nutshell. Princeton University Press, Princeton.

5. Lewis, G.F. (2020) Gravitational Microlensing Time Delays at high Optical Depth: Image Parities and the Temporal Properties of Fast Radio Bursts. Monthly Notices of the Royal Astronomical Society, 497, 1583-1589.

https://doi.org/10.1093/mnras/staa2044

6. Goyal, A., Stawarz, L., Ostrowski, M., et al. (2017) Multiwavelength Variability Study of the Classical BL Lac Object PKS 0735+178 on Timescales Ranging from Decades to Minutes. The Astrophysical Journal, 837, 127. https://doi.org/10.3847/1538-4357/aa6000

7. Mao, S. and Loeb, A. (2001) Gravitational Microlensing of Gamma-Ray Burst Afterglows by Single and Binary Stars. The Astrophysical Journal Letters, 547, L97. https://doi.org/10.1086/318912

8. Wyithe, J.S.B. and Turner, E.L. (2002) Cosmological Microlensing Statistics: Variability Rates for Quasars and Gamma-Ray Burst Afterglows and Implications for Macrolensing Magnification Bias and Flux Ratios. The Astrophysical Journal, 575, 650. https://doi.org/10.1086/341481

9. Vedantham, H.K., Readhead, A., Hovatta, T., et al. (2017) Symmetric Achromatic Variability in Active Galaxies: A Powerful New Gravitational Lensing Probe?. The Astrophysical Journal, 845, 89.

https://doi.org/10.3847/1538-4357/aa745c 